\title{
Smart home automation and monitoring system
}

\author{
A. P. Vancea \\ Electric, Electronic and Computer Engineering, Technical \\ University of Cluj-Napoca, \\ North University Center Baia Mare, Baia Mare, Romania \\ alexvancea92@gmail.com
}

\begin{abstract}
Our project describes a system for the automation and monitoring of a smart house. The system consists of several sensors such as: temperature sensor, humidity sensor, light sensor and presence sensor. The data from sensors is processed and transmitted to the central module via Xbee-ZigBee or to a smartphone through the Bluetooth module. The central module connects at the Internet via $\mathrm{WiFi}$ and through an application we can remotely monitor the state of the house or control various devices within the enclosure.
\end{abstract}

Keywords: sensors, wireless communication, smartphone, internet, smart house;

\section{I.INTRODUCTION}

At present the importance of automation and computerization in all fields is becoming increasingly important. A home equipped with state-of-the-art automated systems should simplify our life and not complicate it. In most cases, the goal of improving technologies is to protect people and provide them with the necessary comfort. Therefore, integrating intelligent equipment into a home infrastructure simply simplifies our life and saves our time and money indirectly.

An intelligent home is equipped with multiple power management systems, home maintenance costs, electronic payment systems, various monitoring modes, and so on. Automation makes the home a practical and easy to use multifunctional complex, harmoniously and coherently integrating the whole home audio and video system, communications, air conditioning, lighting, security, the internet, etc.

Studying several papers in this field, we found that several types of sensors are used to monitor smart house parameters. The most used sensors for monitoring smart home parameters are: light sensor, glass break sensor, gas detector, motion sensor, beam sensor, freeze sensor, smoke sensor, water sensor, humidity sensor, presence sensor and camera [1-6]. An important part of these smart home monitoring systems is their remote accessibility and the possibility to intervene in an emergency. For the connectivity of smart home monitoring and automation
I. Orha

Electric, Electronic and Computer Engineering, Technical University of Cluj-Napoca,

North University Center Baia Mare, Baia Mare, Romania ioan.orha@cunbm.utcluj.ro

systems are used technologies such as: Internet connectivity, GSM technology $[1,2,8]$ or wireless connectivity $[5,7,9]$ Smart home security is a very useful application of IoT and it is used to create an inexpensive security system for homes as well as industrial use [9].

\section{SYSTEM DESCRIPTION}

Our system, presented in Fig.1, is built to acquire data from different sensors (temperature, humidity, light intensity, presence detection, and accelerometer). The data is processed, stored on an SD Card and transmitted via ZigBee wireless communication to central module. Via Bluetooth transmission, you can view in real-time the data from modules on smartphones, tablets, or send infrared (IR) instructions to control home appliances (DVD, TV, Climate).With the data provided by the accelerometer we have the possibility to detect the abnormal state (free fall, earthquake). The central module connects to the Internet via $\mathrm{WiFi}$ and through the dedicated application we can remotely monitor the state of the home or control various devices within the enclosure.

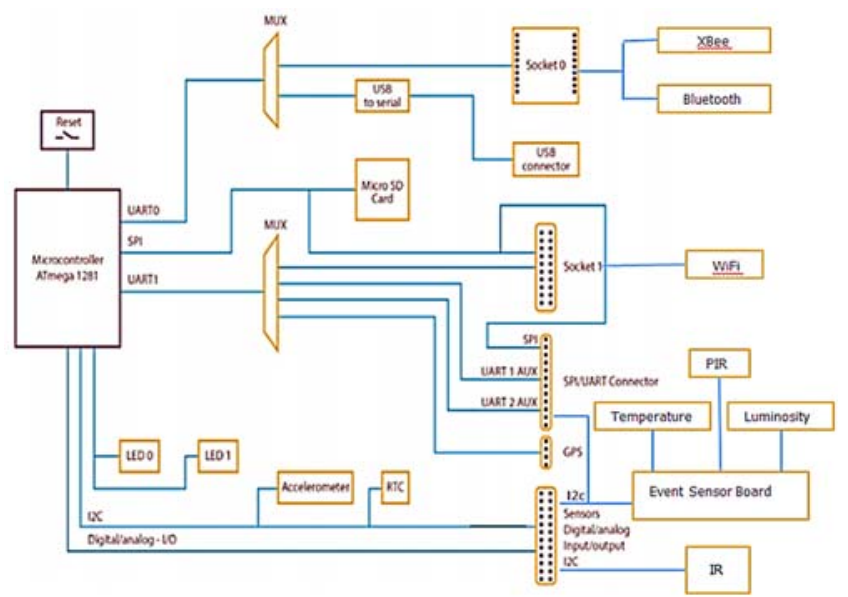

Fig. 1. System structure

Waspmote platform, presented in Fig.2, is an opensource wireless sensor platform specially focused on the implementation of low power modes to allow sensor nodes to be completely autonomous, offering a variable service life of 
between 1 and 5 years depending on the operating cycle and the radio frequency used. Waspmote can connect through 10 different radio frequency protocols: 802.15.4, ZigBee, WiFi, Bluetooth, GPRS, 3G, RFID, NFC, 868MHz, and 900MHz.

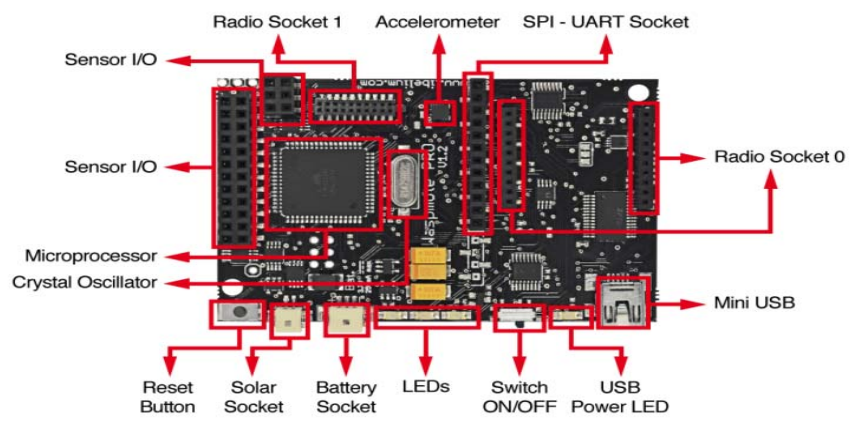

Fig.2. Waspmote platform

The idea was to use the same type of wireless communication for greater compatibility with other devices, so new RF modules (such as Wi-Fi, Bluetooth and NFC) have been designed that use the same socket as the original XBee , thus enabling the user to use the preferred communication type.

The sensors are active on the sensory event plate while Waspmote is in idle mode. When a sensor picks up a value higher than a previously programmed threshold, a signal is generated that wakes the board out of its low consumption and tells which sensor has generated the signal.

In Fig.3. is presented the Events sensors board and the switches for each socket. The board allows simultaneous connection with up to 8 sensors whose outputs are compared with a threshold value and are combined into a logic gate OR that implements a change in interrupt bits that awaken the board. The value of these thresholds is programmed by the $\mathrm{I} 2 \mathrm{C}$ microcontroller.

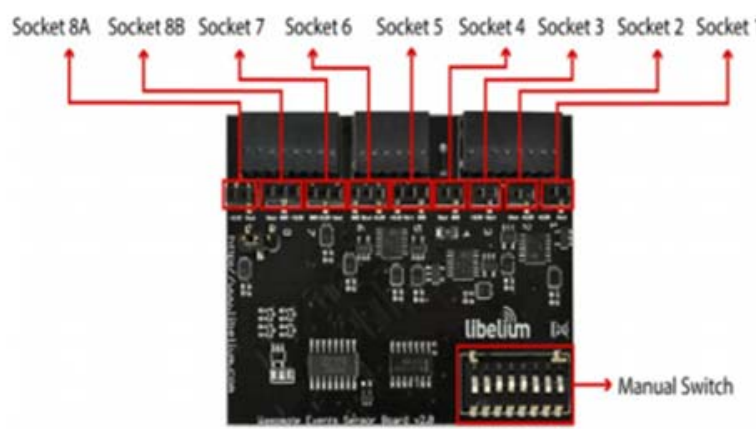

Fig.3. Events Sensors Board

The system consists of several sensors such as: temperature sensor, humidity sensor, light sensor and presence sensor.

Humidity Sensor is an analogue sensor that provides a proportional output to atmospheric relative humidity with an accuracy of $\pm 6 \%$. In Fig. 4 . is presented a code sequence for reading the values from the humidity sensor;

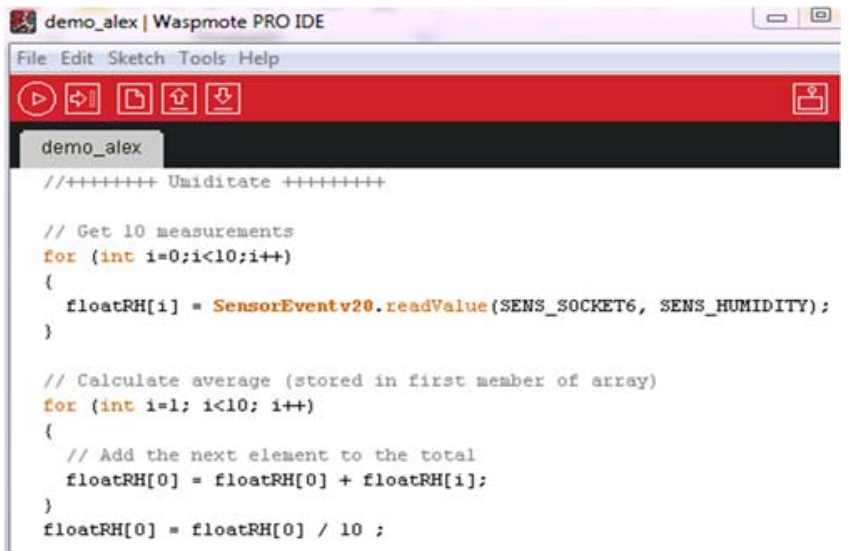

Fig.4. Sequence of humidity reading code

Temperature sensor (MCP9700A) is an analog sensor that converts a temperature value into an analog proportional voltage. Fig.5. illustrates a code sequence used for reading the values from the temperature sensor.

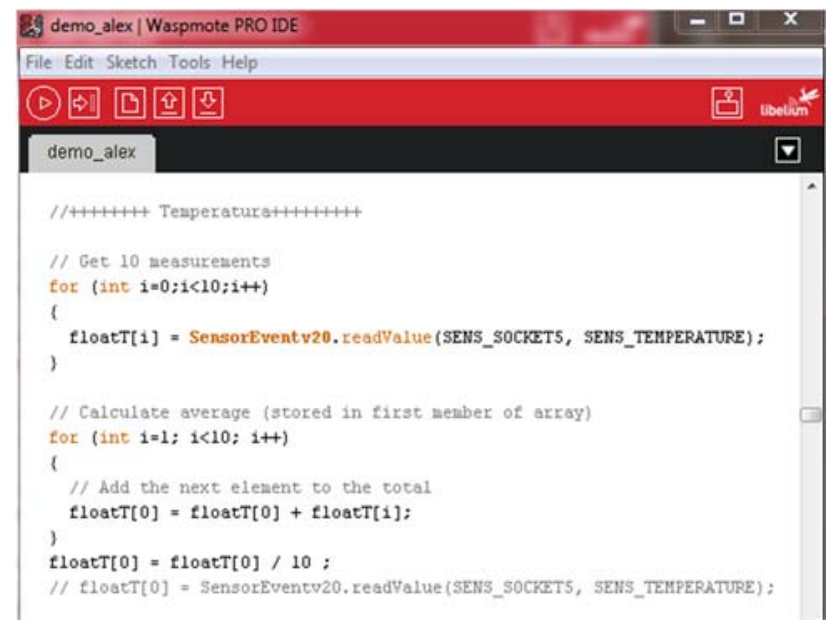

Fig.5 . The sequence of code for temperature sensor

Waspmote has integrated a LIS3331LDH acceleration sensor built by ST Microelectronics that receives the acceleration variations on each of the 3 axes $(X, Y, Z)$. The integration of this sensor allows four kinds of events: free fall, inertial wake, $6 \mathrm{D}$ motion and $6 \mathrm{D}$ position.

The accelerometer communicates with the microcontroller via the $\mathrm{I} 2 \mathrm{C}$ interface.

Waspmote integrates Digi XBee communication modules into ISM (Industrial Scientific Medical). This module communicate with the microcontroller using UART_0 and UART_1 at 115200 bps. This module was chosen for sensitivity and high power transmission/reception. ZigBee is supported in the IEEE 802.15.5 link layer, with the particularity that the XBee-ZBPRO model limits the number of channels to 13 .

The WiFi module for the Waspmote platform complements the current connectivity capabilities that allow the direct communication of sensor nodes with any $\mathrm{WiFi}$ 
router on the market. In addition, this radio allows Waspmote to send information directly to any iPhone or Android smartphone without the need for an intermediate router, making it possible to create WiFi sensor networks anywhere using just Waspmote a mobile device and batteries with this module. Waspmote can make HTTP connections to recover and send information to Web and FTP servers, as well as using TCP / IP and UDP / IP sockets to connect to any server on the Internet.

\section{EXPERIMENTAL MEASUREMENTS}

The implemented system for smart home monitoring proposed by us, as shown in Fig. 6, is fully functional. It allowed to remotely monitor the state of the house or control various devices within the enclosure and recording of data in files on SD CARD.

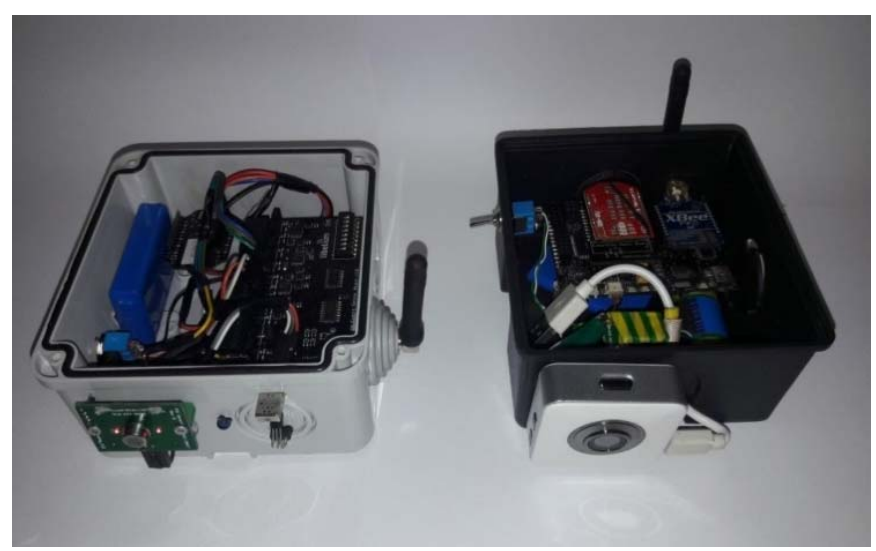

Fig.6. Implemented system for smart home monitoring proposed by us

Fig.7. presents the ZigBee transmission frame. In order to communicate these two modules, the frame must contain the connection information (PAN ID, channel) type and frame structure as shown in the Fig.8.

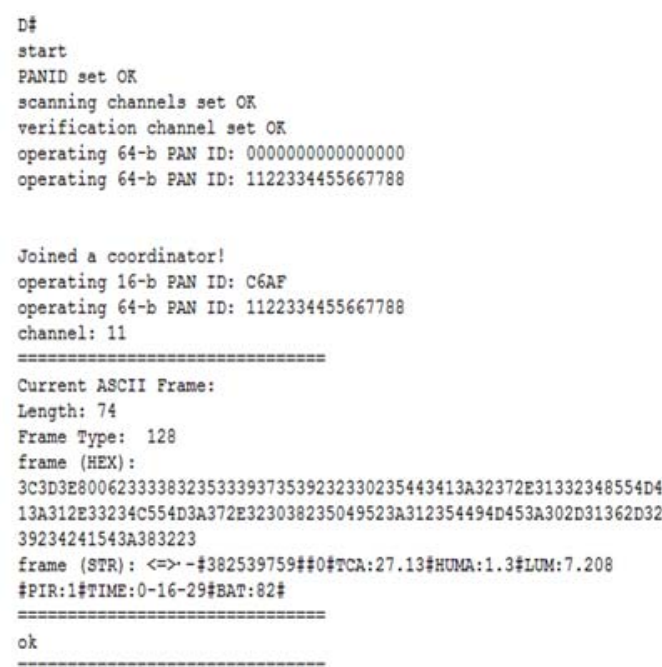

Fig.7. The ZigBee transmission frame

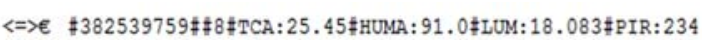
\#TIME: $33-33-0$ \#AT: $63 \# S T R:$ data frame $¥ B A T: 63 \ddagger A C C: 26 ;-42 ; 1046$ \#

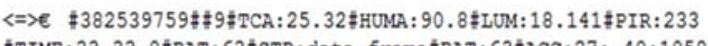

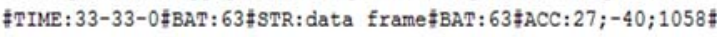

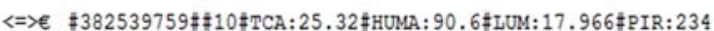

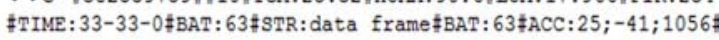

〈>૯ \#382539759\$\#11\#TCA: $25.48 \#$ HUMA : 90.7 LUM: 17.442 \#IR: 234 \#TIME: $33-33-0 \ddagger B A T: 63 \ddagger S T R:$ data frame $\ddagger B A T: 63 \ddagger A C C: 25 ;-42 ; 1055 \$$

« $=>$ \$382539759\$\$12\$TCA: $25.48 \$$ HUMA $: 90.7$ \#UM: $17.093 \$ P I R: 234$

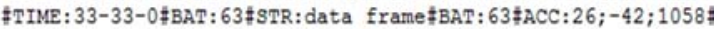

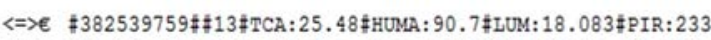
\#TIME: $33-33-0$ \#AT: $63 \# S T R$ : data frame $¥ B A T: 63 \ddagger A C C: 26 ;-40 ; 1060 \ddagger$

〈=> \#382539759\#\#14\#TCA:25.35\#HUMA: $90.7 \#$ LUM: $17.267 \# P I R: 234$

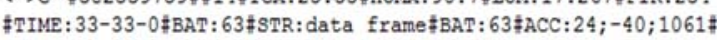

Fig.8. The frame received by the central module.

Fig.9. presents the values from sensors in terminal on the phone via WiFi transmission and Fig.10. present the backup information stored on SD CARD.

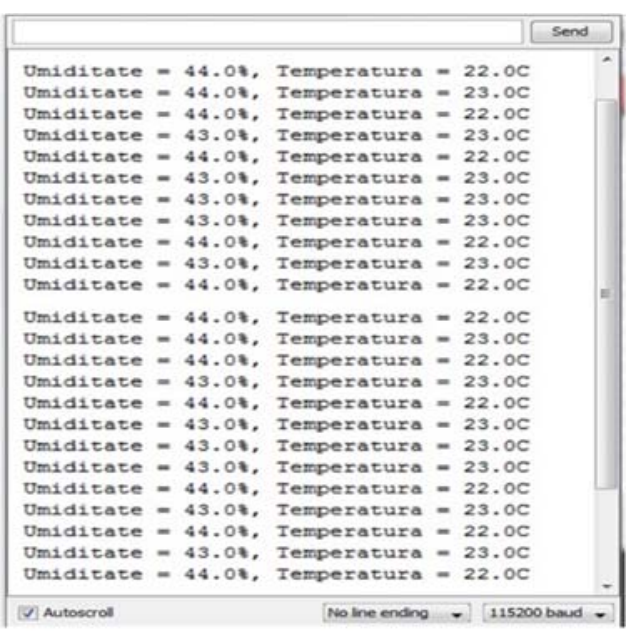

Fig.9. The values from sensors in terminal on the phone via WiFi transmission.

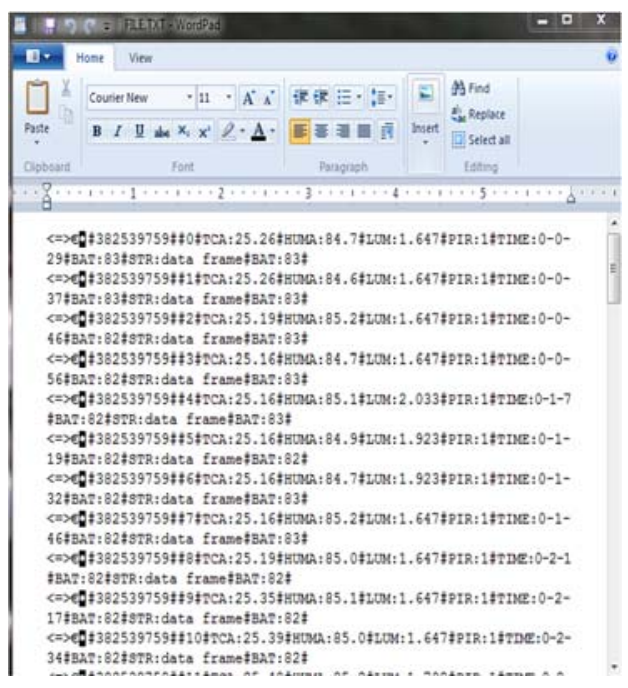

Fig. 10. The backup information stored on SD CARD 


\section{CONCLUSIONS}

The module is functional and provides data reading from sensors, processing and transmitting them to a central module.

The module is independent, has a local power supply (battery) and communicates via Bluetooth with a smartphone, tablet, laptop, etc.

With the help of a smart phone via Bluetooth and Infrared communication we can control the equipment in the premises: TV, DVD, and climate. The central module connects to the Internet via $\mathrm{WiFi}$, and through the dedicated application installed on the smartphone, we can see the data provided by modules;

Power consumption is low, allowing the system to operate for long periods of time due to the state of the "Hibernation".

The module can be improved by attaching sensors for gas detection, fluid level, liquid detection, oxygen quality, bending sensors, pressure, etc.

In the event of transmission failure, the data can be verified due to local storage on the SD card.

With this data we can create databases, graphs on temperature, humidity and brightness in a particular area or room.

\section{REFERENCES}

[1] J. Bangali, A. Shaligram, Design and Implementation of Security Systems for Smart Homebased on GSM technology, International Journal of Smart Home, Vol.7, No.6 (2013), pp.201-208.

[2] E. Isa, N. Sklavos, Smart Home Automation: GSM Security System Design \& Implementation, Journal of Engineering Science and Technology Review 10 (3) (2017) 170-174.
[3] B. Suresh Babu, Dr. C. Venkata Narasimhulu, "Robust Model to Access Consumer Appliances Using Android", International Journal of Research in Advent Technology, Vol.2, No.10, October 2014.

[4] P. Bhagyalakshmi, G. Divya, N.L. Aravinda, "Raspberry PI And Wifi Based Home Automation ”, International Journal of Engineering Research and Applications, pp. 57-60, January 2015

[5] N. Sukumar, A.S. Abhinay, "Web Server Implementation for Embedded Home Automation by Using IP Protocol", Proceedings of International Conference on Emerging Trends in Engineering \& Technology, pp. 147-151, September 2014

[6] C. Sarode, H.S.Thakar, Intelligent Home Monitoring System, International Journal of Engineering Research and Applications (IJERA) ISSN: 2248-9622, Vol. 3, Issue 1, January -February 2013, pp.1446-1450

[7] P. Hamernik, P. Tanuska, Member, IACSIT, and D. Mudroncik, Classification of Functions in Smart Home, International Journal of Information and Education Technology, Vol. 2, No. 2, April 2012

[8] X. Zhenghua , C. Guolong, H. Li , Q. Song, L. Hu, C. Lei , M. Youwen, X. Yexiang, The smart home system based on the iap15f2k61s2 and gsm, international journal on smart sensing and intelligent systems Vol. 7, No. 4, December 2014

[9] A. Anitha, Home security system using internet of things, IOP Conf. Series: Materials Science and Engineering 263 (2017) 042026 doi:10.1088/1757-899X/263/4/042026.

[10] T. Gowthami , G. Adiline Macriga, Smart Home Monitoring and Controlling System Using Android Phone, International Journal of Emerging Technology and Advanced Engineering, Website: www.ijetae.com (ISSN 2250-2459, ISO 9001:2008 Certified Journal, Volume 3, Issue 11, November 2013). 